eISSN 2444-7986

DOI: https://doi.org/10.14201/orl201784.16751

Caso clínico

\title{
MANIFESTACIONES ORALES DE LA SÍFILIS. CASO CLÍNICO
}

\section{Oral manifestations of syphilis. Clinical Case}

\author{
Ana Isabel NAVAZO-EGUÍA ${ }^{1}$; Elena RIOJA-PEÑARANDA ${ }^{1}$; Celina ECHEBARRIA-ITURBE²; \\ Danilo TERÁN-MUÑOZ1 ; Cristina CORDERO-CIVANTOS ${ }^{1}$; Cristina IBAÑEZ-MUÑOZ ${ }^{1}$
}

\author{
${ }^{1}$ Servicio de Otorrinolaringología. ${ }^{2}$ Servicio Anatomía Patológica. Hospital Universitario de Burgos. Burgos. España. \\ Correspondencia: navazoeguia@gmail.com
}

Fecha de recepción: 1 de julio de 2017

Fecha de aceptación: 18 de agosto de 2018

Fecha de publicación: 20 de agosto de 2018

Fecha de publicación del fascículo: 1 de diciembre de 2017

Conflicto de intereses: Los autores declaran no tener conflictos de intereses

Imágenes: Los autores declaran haber obtenido las imágenes con el permiso de los pacientes

Política de derechos y autoarchivo: se permite el autoarchivo de la versión post-print (SHERPA/RoMEO)

Licencia CC BY-NC-ND. Licencia Creative Commons Atribución-NoComercial-SinDerivar 4.0 Internacional

Universidad de Salamanca. Su comercialización está sujeta al permiso del editor

RESUMEN

Introducción: La sífilis es una enfermedad de transmisión sexual causada por la bacteria Treponema pallidum. Las manifestaciones orales de la sífilis no son frecuentes, pero pueden presentarse en todos sus estadios simulando otras enfermedades, retrasando el diagnóstico y tratamiento. Describimos el caso de un paciente con lesiones orales como manifestación de una sífilis secundaria. Discusión: las manifestaciones orales de la sífilis pueden simular otras lesiones orales más comunes, lo que dificulta y retrasa el diagnóstico y tratamiento correcto. Aunque las lesiones orales pueden manifestarse en todas las etapas se asocian principalmente con la sífilis secundaria. El diagnóstico definitivo se realiza generalmente mediante los tests serológicos, sin embargo, se precisa la sospecha clínica e histológica. Conclusión: Dada la creciente incidencia de la sífilis, representa un problema de salud pública y debe tenerse en cuenta en el diagnóstico diferencial de las lesiones orales en los pacientes sexualmente activos.

PALABRAS CLAVE sífilis; manifestaciones orales; Treponema pallidum

SUMMARY Introduction: Syphilis is a sexually transmitted disease caused by the bacterium Treponema pallidum. Oral manifestations of syphilis are not frequent but may occur in all stages simulating other diseases, delaying diagnosis and treatment. We describe the case of a patient with oral lesions as a manifestation of secondary syphilis. Discussion: oral manifestations of syphilis may mimic other more common oral mucosa lesions, going undiagnosed and with no proper treatment. Although oral lesions may manifest at all stages, are mainly associated with secondary syphilis. The diagnosis is usually made through serologic tests, however that suspicion needs to be maintained by the clinical and histologic findings. Conclusion: Given the increasing incidence of syphilis, it remains a public health problem worldwide and should be considered in the differential diagnosis of oral lesions in sexually active patients.

KEYWORDS syphilis; oral manifestations; Treponema pallidum 


\section{INTRODUCCIÓN}

La sífilis es una enfermedad bacteriana producida por la espiroqueta Treponema pallidum. Se le conoce como «la gran simuladora» pues las manifestaciones sistémicas o locales a lo largo de sus diferentes etapas imitan otras enfermedades [1, 2]. La transmisión se produce principalmente a través de relaciones sexuales no protegidas y aunque el sitio principal de inoculación son los órganos genitales, áreas extragenitales como la cavidad oral pueden verse afectadas. La enfermedad progresa a través de diferentes etapas [1-3]. La sífilis primaria afecta a la piel o las membranas mucosas. La lesión clásica es una úlcera no dolorosa de bordes elevados (chancro) que se localiza en la zona del contacto, y que aparece generalmente de 3 a 12 semanas tras la inoculación, frecuentemente seguido de una linfadenopatía regional no sensible. Los chancros duran de 3 a 6 semanas y se curan con independencia del tratamiento. La sífilis secundaria se manifiesta 4 a 6 semanas después de la desaparición del chancro y expresa la propagación hematógena de Treponema pallidum. La manifestación más característica es una erupción maculo-papulosa generalizada de tronco y extremidades, así como palmas y plantas (roséola sifilítica). La erupción puede afectar a las mucosas con lesiones con pápulas sobreelevadas grisáceas o blanquecinas, llamados condilomas planos. La sífilis latente, sigue a la desaparición de las lesiones de la sífilis primaria y secundaria. En esta etapa la infección continua en los pacientes no tratados y puede durar años. Este período se divide, a su vez, en latente precoz y en latente tardío (más de 12 meses). La recaída de una sífilis secundaria es más probable en esta fase precoz y se produce como consecuencia de una disfunción inmunológica. La sífilis tardía, suele aparecer después de 10 a 30 años de haberse contagiado. Se caracteriza por manifestaciones $\mathrm{Cu}-$ taneomucosas (gomas superficiales y profundas) $y$, por afectaciones viscerales (cardiovasculares o neurológicas).

El objetivo de este artículo es presentar el caso de un paciente con manifestaciones orales en el curso de una sífilis secundaria.

\section{DESCRIPCIÓN}

Paciente varón de 35 años, remitido por presentar múltiples lesiones ulcerosas indoloras en paladar blando y borde lingual y papilomatosas en ambas regiones amigdalares (Figura 1).
En el momento de la exploración no reconoció antecedentes patológicos previos ni relaciones de riesgo. No presentaba otras lesiones mucocutáneas.

Se realizó biopsia con resultado de intensa inflamación constituida fundamentalmente por células plasmáticas y positividad inmunohistoquímica para treponemas (Figuras 2 y 3 ). Virus del papiloma humano negativo negativo.

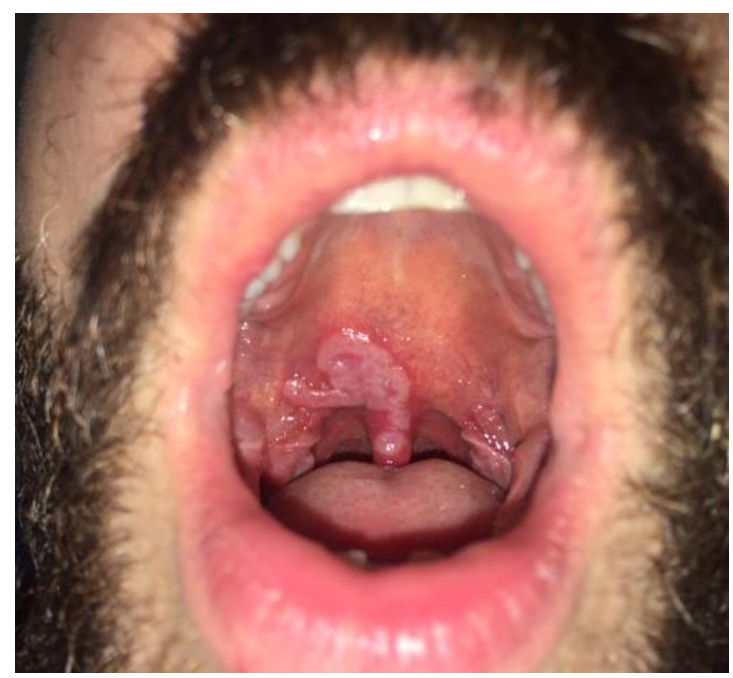

Figura 1. Lesiones ulcero-papulosas en paladar blando y ambas regiones amigdalares.

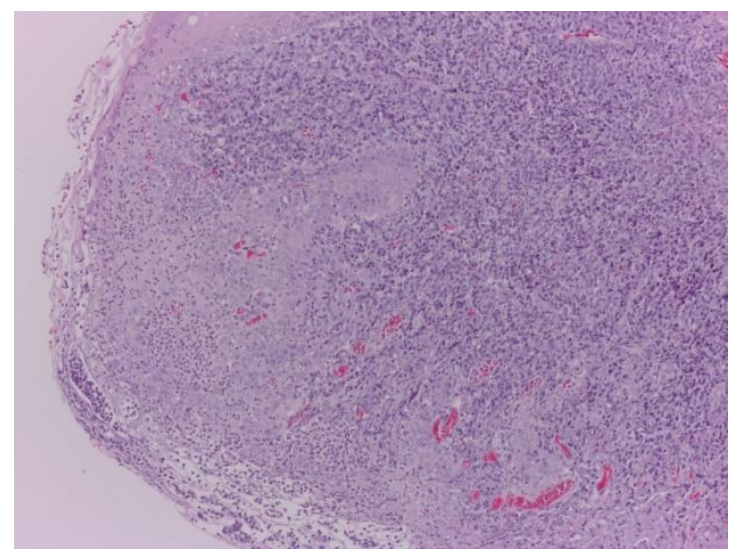

Figura 2. Mucosa oral de morfología papilomatosa con hiperplasia del epitelio y un intenso infiltrado inflamatorio con presencia de abundantes células plasmáticas (HE 100x).

Reevaluado el paciente, reconoció prácticas de sexo oral con varones no protegidas y, un cuadro de exantema rosáceo en las palmas de las manos 2 años antes, que fue diagnosticado de sífilis secundaria y tratado con una dosis de 
penicilina $\mathrm{G}$ benzatina, sin seguimiento posterior.

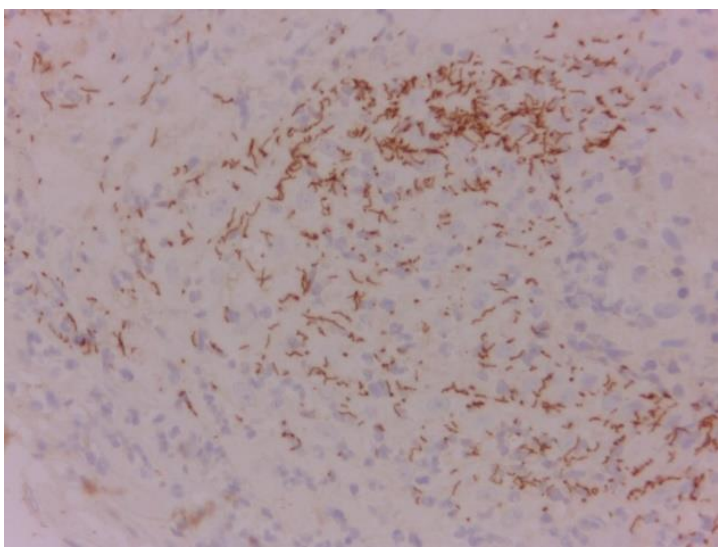

Figura 3. Presencia de abundantes bacilos positivos en estudio inmunohistoquímico con anticuerpo para detección de Treponema pallidum (400x).

Se realizó estudio serológico para $\mathrm{VIH}$ negativo, RPR (+) 1/16 y TPHA (+) 1/5120. Anticuerpos totales para sífilis positivos.

Se instauró tratamiento con penicilina $G$ benzatina $(2.400 .000 \mathrm{UI})$ una dosis semanal durante 3 semanas, con resolución de las lesiones orales, y se remitió al servicio de enfermedades infecciosas para su seguimiento y descartar otras ETS asociadas.

\section{DISCUSIÓN}

La incidencia de la sífilis ha ido aumentando en todo el mundo [4, 5], ocurriendo principalmente entre hombres homosexuales, siendo frecuente la coincidencia con otras enfermedades de transmisión sexual como el VIH y hepatitis.

Las presentaciones atípicas de la sífilis representan un reto diagnóstico especialmente para médicos no habituados o en países con baja incidencia lo que incrementa las consecuencias de una terapia tardía o inadecuada, y un mayor riesgo de transmisión del $\mathrm{VIH}$ [4-6].

Por estas razones, debemos estar familiarizados con las manifestaciones clínicas de la sífilis.

La presentación puede ser atípica y afectar a la cavidad oral generalmente en pacientes que practican el sexo oral sin protección [7, 8].

Clínicamente, la manifestación oral de la sífilis puede parecerse a otras entidades, lo que dificulta el diagnóstico correcto por lo que debe tenerse en cuenta en el diagnóstico diferencial de las lesiones ulcerosas orales en los pacientes sexualmente activos [7, 9].

La sífilis primaria puede observarse en la cavidad oral, y se ha asociado con el sexo oral [10, 11]. El chancro de inoculación de la sífilis primaria puede ocurrir en la boca y región perioral pero es infrecuente (sólo $8 \%$ de los casos) [12-15].

Aunque las lesiones orales pueden manifestarse en todas las etapas se asocian principalmente con la sífilis secundaria. Se observan manifestaciones orales en el $30 \%$ de los casos de los pacientes, pero rara vez como únicas manifestaciones de la infección [16-19]. Se caracterizan por lesiones eritematosas, erosivas, papulosas o ulcerosas, incluso vegetantes.

Las lesiones de la sífilis primaria y secundaria son altamente contagiosas.

El goma es la lesión oral característica de la sífilis terciaria, es causada por endarteritis obliterante de los vasos, particularmente las arteriolas, dentro de la mucosa y afecta al paladar duro, la lengua o las amígdalas. La glositis intersticial se caracteriza por la atrofia del dorso lingual como consecuencia de una endarteritis obliterativa de los vasos linguales, estas lesiones favorecen la aparición de leucoplasias y carcinomas [1-3].

Un $25 \%$ de pacientes tienen episodios recurrentes de sífilis secundaria fundamentalmente en el primer año durante la fase latente precoz, que puede manifestarse exclusivamente en la mucosa oral como en el caso que presentamos [20].

La etapa secundaria de la enfermedad toma una variedad de manifestaciones orales, imitando otras lesiones más frecuentes en la cavidad oral, permaneciendo sin tratamiento adecuado [16-22]. El análisis de la historia clínica de un paciente sospechoso, combinado con el examen físico y los exámenes serológicos, normalmente proporcionan un diagnóstico concluyente de la enfermedad, y la biopsia no se requiere normalmente como un diagnóstico inicial [22].

Las pruebas serológicas siguen siendo el pilar del diagnóstico de la sífilis [22-24]. Lo tradicional es utilizar una prueba serológica no treponémica (VDRL o RPR) para el cribado seguido de una prueba serológica específica (TPHA, FTA-ABS) de antígeno treponémico para la confirmación si la prueba de detección es positiva.

La penicilina es el fármaco de elección para el tratamiento de todas las etapas de la sífilis 
[25]. El tratamiento será una dosis intramuscular de 2.400.000 UI de penicilina G benzatina en la etapa primaria. Sin embargo, en la etapa secundaria y etapa terciaria la misma dosis se repite semanalmente durante tres semanas.

Para los pacientes alérgicos a la penicilina, se indica la administración oral de doxiciclina 100 mg dos veces al día durante 14 días o 500 mg de tetraciclina cuatro veces al día durante 14 días.

\section{CONCLUSIONES}

El diagnóstico de la sífilis es un desafío ya que imita a otras enfermedades. El pronóstico de estos los pacientes está directamente relacionado con un diagnóstico precoz y la eficacia del tratamiento. Dada la creciente incidencia de la sífilis, debe tenerse en cuenta en el diagnóstico diferencial de las lesiones orales. Es importante la derivación a un servicio de enfermedades infecciosas dada la necesidad de descartar otras enfermedades de transmisión sexual asociadas.

\section{BIBLIOGRAFÍA}

1. Larsen SA. La sífilis en el momento actual. En: Picazo JJ, Bouza E (eds). Infección 1999. Servisistem 2000 SL, Bilbao, 1999, pp 177-216.

2. Castañeda Curto $N$, Obeso Agüera $S$, Morales Angulo C. Patología orofaríngea secundaria a contacto orogenital. Rev Soc Otorrinolaringol Castilla León Cantab La Rioja. [Internet] 2014 [citado el 27 de junio de 2017]; 5:85-7. Disponible en: http://gredos.usal.es/jspui/handle/10366 1124038 .

3. Hicks CB, Sparling PF. Pathogenesis, clinical manifestations, and treatment of early syphilis. 2013. In: UpToDate, Post TW (Ed), UpToDate, Waltham, MA. [Citado el 27 Jun. 2017].

4. Centers for Disease Control and Prevention. Sexually Transmitted Disease Surveillance [Internet] 2013. Atlanta: U.S. Department of Health and Human Services; 2014. Disponible en https://www.cdc.gov/std/stats13/surv20 13-print.pdf [Citado el 27 de junio de 2017].
5. Tucker JD, Yin YP, Wang B, Chen XS, Cohen MS. An expanding syphilis epidemic in China: epidemiology, behavioural risk and control strategies with a focus on low-tier female sex workersand men who have sex with men. Sex Transm Infect. 2011;87(Suppl 2):16-8.

6. Yang CJ, Chang SY, Wu BR, Yang S4, Liu WC, Wu PY, et al. Unexpectedly high prevalence of Treponema pallidum infection in the oral cavity of human immunodeficiency virus-infected patients with early syphilis who had engaged in unprotected sex practices. Clin Microbiol Infect. 2015;21(8):787.e17.

7. Ikenberg K, Springer E, Brauninger W, Kerl K, Mihic D, Schmid S, et al. Oropharyngeal lesions and cervical lymphadenopathy: syphilis is a differential diagnosis that is still relevant. J Clin Pathol. 2010;63:731-6.

8. Fernández-López $\mathrm{C}$, Morales-Angulo C. Lesiones otorrinolaringológicas secundarias al sexo oral. Acta Otorrinolaringol Esp. 2017;68(3):16980.

9. Dybeck Udd S, Lund B. Oral Syphilis: A Reemerging Infection Prompting

1. Clinicians' Alertness. Case Rep Dent. 2016;2016:6295920.

10. Fregnani ER, Pérez-de-Oliveira ME, Parahyba CJ, Perez DE. Primary syphilis: An uncommon manifestation in the oral cavity. J Formos Med Assoc. 2017;116(4):326-7.

11. Bjekić M, Marković M, Sipetić S. Clinical manifestations of primary syphilis in homosexual men. Braz J Infect Dis. 2012;16(4):387-9.

12. Drago F, Ciccarese G, Cogorno L, Tomasini CF, Cozzani EC, Riva SF et al. Primary syphilis of the oropharynx: an unusual location of a chancre. Int J STD AIDS. 2015;26(9):679-81.

13. Gedela K, Boag F. Syphilic tonsillitis in primary care: a case report. Brit J Gen Pract. 2012:219-20. 
14. Hertel M, Matter D, SchmidtWesthausen AM, Bornstein MM. Oral syphilis: a series of 5 cases. J Oral Maxillofac Surg. 2014;72(2):338-45.

15. Leuci S, Martina S, Adamo D, Ruoppo E, Santarelli A, Sorrentino R, et al. Oral Syphilis: a retrospective analysis of 12 cases and a review of the literature. Oral Dis. 2013;19(8):738-46.

16. Ortega KL, Rezende NP, Watanuki F, Araujo NS, Magalhaes MH. Secondary syphilis in an HIV positive patient. Med Oral 2004;9:33-8.

17. Seibt CE, Munerato MC. Secondary syphilis in the oral cavity and the role of the dental surgeon in STD prevention, diagnosis and treatment: a case series study. Braz J Infect Dis. 2016;20(4):3938.

18. Hamlyn E, Marriott D, Gallagher RM. Secondary syphilis presenting as tonsillitis in three patients. J Laryngol Otol. 2006;120:602-4.

19. de Paulo LF, Servato JP, Oliveira MT, Durighetto AF, Zanetta-Barbosa D. Oral Manifestations of Secondary Syphilis. Int J Infect Dis. 2015;35:40-2.

20. Carlesimo M, Palese E, Mari E, Feliziani G, La Pietra M, De Marco G, et al. Isolated oral erosions: an unusual manifestation of secondary syphilis. Dermatol Online J. 2008;14(2):23.
21. Carbone PN, Capra GG, Nelson BL. Oral Secondary Syphilis. Head Neck Pathol. 2016;10:206-8.

22. Strieder LR, León JE, Carvalho YR, Kaminagakura E. Oral syphilis: report of three cases and characterization of the inflammatory cells. Ann Diagn Pathol. 2015;19:76-80.

23. Larsen SA, Steiner BM, Rudolf $\mathrm{AH}$. Laboratory diagnosis and test for syphilis. Clin Microbiol Rev. 1995;8:121.

24. Siqueira CS, Saturno JL, de Sousa SC, da Silveira FR. Diagnostic approaches in unsuspected oral lesions of syphilis. Int J Oral Maxillofac Surg 2014;43(12):1436-40.

25. Workowski KA, Berman S, Centers for Disease Control and Prevention (CDC). Sexually transmitted diseases treatment guidelines. MMWR. 2010. Sex Transm Dis. 2012; 39(12):954-8.

\section{ENLACES RELACIONADOS}

Centers for Disease Control and Prevention (CDC). https://www.cdc.gov/ 